length of life of small mammals. Criticism is levelled at the practice of assuming exponential population growth in certain species and different methods of estimating longevity are compared.

The final section on energy flow and productivity estimations contains six papers. It is interesting to note that although a bank vole has an average life expectancy of up to 3.66 months it is clear that some 60 per cent of its production occurs within the first 0.75 months of its life. This underlines the necessity of finding ways to study what is normally regarded as a non-trappable part of the population. The same conclusion is reached in another paper which deals with the field vole. The remaining papers are primarily concerned with food consumption by small rodents and it would appear that these animals rarely eat more than 1 to 2 per cent of the primary production potentially available to them. This suggests that food might not be a limiting factor to the size of some populations, although one author is careful to point out that food might become a critical factor which limits those overwintering populations which follow a peak in vole numbers.

Clearly, this volume summarizes a wealth of information about diverse matters relating to energy flow through small mammal populations. Typographical errors occur frequently in the text but in no instance did I find them particularly misleading and therefore I have refrained from detailing them. In conclusion it must be said that any mammalogist or ecologist professing an interest in small mammal population density estimates, production or energy flow would be doing himself a disservice if he did not consult this publication.

John Phillipson havioural science, especially in its scientific and research aspects, and, hopefully, to "constitute a basis for an informed, effective national policy to strengthen and develop" these fields. With the public view of psychiatry dominated by the images of the couch and the asylum, the treatment of neurotic private patients by psychoanalysts, and the confinement of psychotic patients in large mental hospitals, there is certainly room for an entertaining and stimulating review of the areas of expansion in modern psychiatry.

Although this volume provides a fairly wide ranging survey of the field of recent and current research in psychiatry, the survey is patchy and by no means as up to date as one expects from a work published in 1970-much of it could have been written five years ago or more. Psychoanalysis is perhaps over-represented for a study of behavioural science; and the emphasis is almost entirely on American work. The presentation is terse and irregular, and manages to be too specific and technical for the lay reader in many passages, as well as too vague and lacking in detail for the professional reader. There is an intelligent and reasonably selective list of annotated references.

The style of writing is turgid and uniformly woolly, which is unpardonable when dealing with such a lively and potentially exciting subject. The layout and design are singularly uninspiring, and the long slabs of grey type are not always easy to read. Perhaps the major weakness of the book is the common one of aiming at a great many targets and missing most of them. It is neither sufficiently clear and attractive to interest and inform many members of the informed public, nor sufficiently critical and specific to be really helpful in guiding future research and developments in psychiatry.

Michael A. Simpson background of non-critical optical transitions from the smaller contribution due to the critical points, which are nevertheless essential in the adjustment of theoretical band structures.

The contributions by Button and Kaplan on spectroscopy at sub-millimetre and far infrared wavelengths, respectively, bear on semiconductor problems. The use of high frequency photons to excite cyclotron resonance or some other transition between allowed electron states makes possible an order of magnitude increase of resolution compared with that available at microwave frequencies and allows the application of Fourier spectroscopy and the use of resonant photoconductivity as a detection system.

The optical properties of the alkali halides and the inert gases seem to be the simplest possible, and several papers in these conference proceedings concentrate on these materials. Optical properties are evidently better understood than for semiconductors. A paper by Zawadzki develops the analogy between free relativistic electrons and electrons in a narrow gap semiconductor, applying the ideas to the behaviour of electrons in crossed electric and magnetic fields and especially to the optical properties of semiconductors under such conditions. Moriya's review of optical excitation of magnons in spin systems describes a field of growing interest rather sketchily and with no experimental details, only references to the original literature.

Such papers draw attention to the conflict between the more conventional review literature and conference proceedings, in that the inadequacies of some of the reviews in this publication force the reader back to the original literature at an earlier stage than is usual in the study of a review. If the excessive cost of this book is set beside its value to the physicist, it is probably not a book to own but one to be borrowed from a library.

Alexander D. C. Grassie

\section{Scientific Psychiatry}

Psychiatry as a Behavioral Science. Edited by David A. Hamburg. (The Behavioral and Social Sciences Survey: Psychiatry Panel.) Pp. xii +114. (Prentice-Hall: Englewood Cliffs, New Jersey, 1970.) \$5.95.

SOMEONE once described a camel as a horse that had been designed by a committee. A book written by a committee starts off at a similar disadvantage. This work, one of a series of reports for the public initiated by the US National Academy of Science and the Social Science Research Council, was prepared by a distinguished panel of American scientists. It aims to provide a comprehensive review of psychiatry as a be-

\section{Poor Substitute}

Optical Properties of Solids. Edited by E. D. Haidemenakis. (Lectures and Seminars presented at the Fifth Chania International Conference, held at Chania, Crete, Greece, June 30-July 10, 1969.) Pp. $x v+520$. (Gordon and Breach: New York and London, November 1970.) $£ 12.25 ; \$ 9.50$.

THIs publication covers optical studies in the alkali halides, the inert gases, semiconductors and magnetic insulators but gives only slight coverage to optical studies of metals. Of especial interest is the review by Seraphin of the use of modulated reflectance in the study of band structures; he shows how the technique can be used to separate the massive

\section{Analysing Proteins}

\section{Protein Sequence Determination:} Methods and Techniques. By S. Blackburn. Pp. ix + 292. (Marcel Dekker: New York, September 1970.) £8; $\$ 16.75$.

THIs excellent account of protein sequence analysis will be useful to chemists, biochemists, molecular biologists and others who require a practical guide to the subject. Automated analysis is still very much in its infancy and, as the author points out, sequencing a protein is not yet a routine operation, each protein presenting a problem in its own right. In twenty chapters the sequencing strategy is revealed with the enthusiasm to be expected from an experienced worker in the field. 